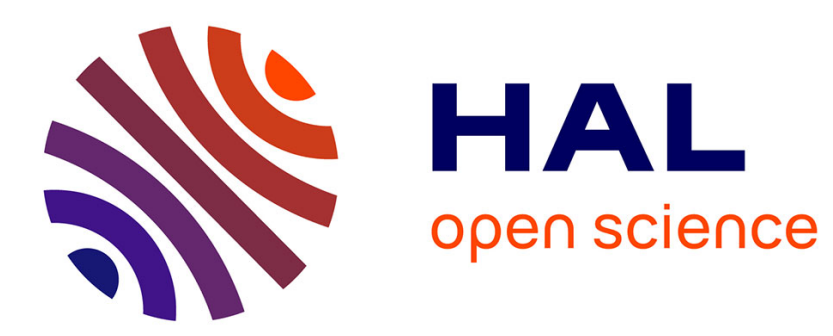

\title{
Driving force for the electromigration of an impurity in a homogeneous metal
}

L. Turban, P. Nozières, M. Gerl

\section{To cite this version:}

L. Turban, P. Nozières, M. Gerl. Driving force for the electromigration of an impurity in a homogeneous metal. Journal de Physique, 1976, 37 (2), pp.159-164. 10.1051/jphys:01976003702015900 . jpa-00208398

\section{HAL Id: jpa-00208398 https://hal.science/jpa-00208398}

Submitted on 1 Jan 1976

HAL is a multi-disciplinary open access archive for the deposit and dissemination of scientific research documents, whether they are published or not. The documents may come from teaching and research institutions in France or abroad, or from public or private research centers.
L'archive ouverte pluridisciplinaire HAL, est destinée au dépôt et à la diffusion de documents scientifiques de niveau recherche, publiés ou non, émanant des établissements d'enseignement et de recherche français ou étrangers, des laboratoires publics ou privés. 


\title{
DRIVING FORCE FOR THE ELECTROMIGRATION OF AN IMPURITY IN A HOMOGENEOUS METAL
}

\author{
L. TURBAN $\left({ }^{*}\right)$, P. NOZIÈRES $\left({ }^{* *}\right)$, M. GERL $\left({ }^{*}\right)$
}

(Reçu le 24 septembre 1975, accepté le 6 octobre 1975)

\begin{abstract}
Résumé. - Par une analyse microscopique, nous montrons que les deux définitions de la valence efficace, l'une en termes de flux de charge associé au déplacement du défaut, l'autre en termes de force agissant sur l'impureté, sont équivalentes.

D'autre part, en utilisant la thermodynamique des processus irréversibles, nous montrons que la force sur une impureté est proportionnelle à sa résistivité spécifique dans la matrice, quel que soit le potentiel d'impureté.

Abstract. - Through a microscopic analysis the thermodynamic definition of the effective valence as the flux of electric charge associated with a unit flux of the solute is shown to be equivalent to the usual definition in terms of the force acting on the impurity in an electric field.

Using the thermodynamics of irreversible processes, we show that the force acting on an impurity is proportional to its specific resistivity in the host metal, whatever the strength of the impurity potential.
\end{abstract}

1. Introduction. - When a direct electric current is passed through a solid solution, the drift velocity of the solute is given by the Nernst-Einstein relation [1]

$$
\mathbf{v}_{\mathbf{i}}=\frac{D_{\mathbf{i}}}{k T} Z_{\mathbf{i}}^{*}|e| \mathbf{E}
$$

where $\mathbf{E}$ is the applied electric field, $D_{\mathrm{i}}$ is the diffusion coefficient and $Z_{i}^{*}$ is the effective valence of the solute. Using the principles of the thermodynamics of irreversible processes, it is possible to show that $Z_{\mathbf{i}}^{*}|e|$ is equal to $\left(\mathbf{J} / \mathbf{J}_{\mathbf{i}}\right)_{\mathbf{E}=0}$, the total flux of electric charge (ionic as well as electronic), associated with a unit flux of the impurity, in the absence of an applied electric field :

$$
Z_{\mathbf{i}}^{*}|e|=\left(\frac{\mathbf{J}}{\mathbf{J}_{\mathbf{i}}}\right)_{\mathbf{E}=0}
$$

Although the equivalence between the definitions (1) and (2) follows from Onsager's relations, it is customary to use eq. (1) to calculate $Z_{\mathrm{i}}^{*}$ : an electric field $\mathbf{E}$ is applied to the sample and the force $\mathbf{F}_{\mathrm{i}}=Z_{\mathrm{i}}^{*}|e| E$ on the impurity is calculated. It is usually thought that $\mathbf{F}_{\mathbf{i}}$ can be split up in two parts;

(*) Laboratoire de Physique du Solide. Université de NancyI, C.O. 140, 54037 Nancy-cedex, France.

(**) Institut Laue-Langevin, B.P. 156, Centre de Tri, 38042 Grenoble-cedex, France.
$\mathbf{F}_{\text {es, }}$ the so-called direct electrostatic force which describes the direct action of the field on the impurity, whereas $\mathbf{F}_{\mathrm{f}}$, the friction force, is due to the transfer of momentum from the charge carriers to the impurity:

$$
\mathbf{F}_{\mathrm{i}}=\mathbf{F}_{\mathrm{es}}+\mathbf{F}_{\mathrm{f}} \text {. }
$$

In the interpretation of their results most experimenters assume that $\mathbf{F}_{\text {es }}=Z_{\mathbf{i}}|e| \mathbf{E}$, where $Z_{\mathrm{i}}$ is the true ionic charge, as if the diffusing entity were a bare ionic charge. As for $\mathbf{F}_{f}$, it has been calculated semiclassically by Huntington [2] and Fiks [3] and in quantum mechanics by Bosvieux and Friedel [4] within the Born approximation. In those papers use is made of a Boltzmann equation to describe the statistics of the perturbed electron gas.

More recently, Turban [5], Kumar and Sorbello [6] and Schaich [7] have calculated $\mathbf{F}_{\mathrm{f}}$ through a linear response formalism. All these papers show that $\mathbf{F}_{\mathrm{f}}$ is related to the specific resistivity $\rho_{\mathrm{i}}=\mathrm{d}(\Delta \rho) / \mathrm{d} n_{\mathrm{i}}$, where $\Delta \rho$ is the resistivity change of an initially pure sample when it dissolves $n_{\mathrm{i}}$ impurities per unit volume :

$$
\mathbf{F}_{\mathrm{f}}=-n_{0} \frac{\rho_{\mathrm{i}}}{\rho}|e| \mathbf{E}
$$

In this expression, $n_{0}$ is the number of conduction electrons per unit volume and $\rho=\rho_{0}+\rho_{\mathrm{i}} n_{\mathrm{i}}$ is the resistivity of the alloy.

The aims of the present paper are (i) to demonstrate the validity of (4) for an arbitrary strength of the 
electron impurity interaction, and (ii) to show that the electrostatic force vanishes, the effect of the electric field on the ion being cancelled by that on the accompanying screening cloud. We shall carry out the analysis in the simple case of an impurity moving in an homogeneous system, like a liquid metal. The extension to real crystals raises new problems due to the fact that the force depends on the position of the impurity in the unit cell (it varies during the hopping process), hence a difference between interstitial and substitutional impurities, etc. In order to make our formulation as transparent as possible, we choose to ignore these complications by considering an homogeneous background.

In part 2 of the present paper, we give a simple, rigorous microscopic derivation of the equivalence between (1) and (2) and we establish the validity of (4).

In part 3, we compare these results with a simple phenomenological analysis of the friction effects.

2. Microscopic analysis. - From definition (2) the effective valence is related to the net flux of electric (ionic and electronic) charge associated with the displacement of a solute ion. Starting from microscopic considerations, it is possible to give a general demonstration of this relation and to show why the direct electrostatic force on the impurity vanishes.

2.1 FluX OF ELECTRIC CHARGE INDUCED BY THE DISPLACEMENT OF AN IMPURITY ION. - We consider a unit volume containing one impurity at position $\mathbf{r}(t)$ moving with the velocity $\mathbf{u}=\dot{\mathbf{r}}(\mathrm{t})$ along the $x$-axis, $u$ being much smaller than the Fermi velocity $v_{\mathrm{F}}$. The Hamiltonian of the electron system can be written :

$$
\mathscr{H}(t)=\mathscr{H}_{0}+V[\mathbf{r}(t)]
$$

where $\mathcal{H}_{0}$ contains the kinetic contribution to $\mathscr{H}(t)$ as will as electron-phonon and electron-electron interactions ; $V$ is the electron-impurity interaction potential :

$$
V[\mathbf{r}(t)]=\sum_{l} v\left(\left|\mathbf{r}_{l}-\mathbf{r}(t)\right|\right)
$$

$\mathbf{r}_{l}$ representing the position of the 1 th electron and $v(\mathbf{r})=-Z_{\mathrm{i}} \frac{e^{2}}{r}$ (actually, the exact form of $V$ is irrelevant to our following argument).

Let us define the operators :

$$
X=\sum_{l} x_{l} ; \quad \dot{X}=\sum_{l} \dot{x}_{l} .
$$

The total electron flux can be written as :

$$
J_{\mathrm{e}}=\operatorname{Tr}(\dot{X} \delta \rho)
$$

where $\delta \rho$ is the change of the density matrix induced by the displacement of the impurity. In order to calculate $\delta \rho$, we take advantage of the fact that the velocity $u$ is small : thus the Hamiltonian, although complicated if the interaction potential $V$ is strong, is slowly varying in time. We can therefore use a method employed by Iche and Nozières in another context [8]. If the relaxation of the electron bath were infinitely rapid, the density matrix would correspond to the instantaneous equilibrium :

$$
\rho_{0}(t)=\frac{\mathrm{e}^{-\beta \mathcal{S E}(t)}}{\operatorname{Tr}\left[\mathrm{e}^{-\beta \mathcal{S E}(t)}\right]} .
$$

Because the relaxation rate of the electron system is finite, $\rho$ departs from $\rho_{0}$ :

$$
\rho(t)=\rho_{0}(t)+\delta \rho(t)
$$

where $\delta \rho$ is small as long as $u$ is small, and obeys the dynamic equation :

$$
i \dot{\rho}_{0}+i \delta \dot{\rho}=[\mathfrak{H}, \delta \rho] .
$$

We see that $\delta \rho$ is forced by the time variation of $\rho_{0}$. For low velocities $\delta \dot{\rho}$ in (11) represents in fact a higher order correction so that we can replace (11) by the simpler equation :

$$
i \dot{\rho}_{0}=[\mathscr{H}, \delta \rho]-i \eta \delta \rho .
$$

In writing (12) we added the new term $-i \eta \delta \rho$ with $\eta \rightarrow+0$ in order to define unambiguously the energy denominators that come into the theory. Physically, we can view this term as an infinitesimal external relaxation towards the instantaneous equilibrium, which is enough to dispose of the irreversibility paradox. In real life, relaxation is supposed to occur in the electron system itself.

The solution (12) is straightforward if at each time we use as a basis the eigenstates of the instantaneous Hamiltonian $\mathcal{H}(t)$ (thereby using a rotating basis). The matrix elements of $\delta \rho$ are then found to be :

$$
\delta \rho_{m n}=\frac{v_{m}-v_{n}}{E_{m}-E_{n}} \frac{\dot{H}_{m n}}{i\left(E_{n}-E_{m}\right)-\eta}
$$

where $v_{m}=\left(\rho_{0}\right)_{m m}$ is the occupation number of the level $E_{m}(t)$ corresponding to the eigenvector $|m(t)\rangle$ of $\mathcal{H e}(t)$. In our problem :

$$
\dot{H}=\frac{\partial \mathscr{H}}{\partial t}=\frac{\partial V}{\partial \mathbf{r}} \cdot \dot{\mathbf{r}}=-u F
$$

where $\quad F=-\frac{\partial V}{\partial x}$

is the operator for the $x$-component of the force on the impurity with position $\mathbf{r}(x, y, z)$ and velocity $u=\dot{x}$.

Inserting (13) and (14) into (8) we obtain the elec- 
tron current induced by the displacement of the impurity and :

$$
\begin{aligned}
\left(\frac{J_{\mathrm{e}}}{u}\right)_{\mathbf{E}=0} & =\frac{1}{u} \sum_{m n} \delta \rho_{m n} \dot{X}_{n m} \\
& =-\sum_{m n} \frac{v_{m}-v_{n}}{E_{m}-E_{n}-i \eta} F_{m n} X_{n m},
\end{aligned}
$$

where the equation of motion of the operator $X$ has been used :

$$
\dot{X}_{n m}=i[\mathcal{H}, X]_{n m}=i\left(E_{n}-E_{m}\right) X_{n m} .
$$

We note that the relation (16) is exact, irrespective of the strength of the interaction $V$ between the electrons and the impurity : we only assumed that the velocity $u$ is small. It remains perfectly valid if we take account of the Coulomb interactions between electrons (all these features are imbedded in the eigenstates $|m(t)\rangle)$. Finally, $J_{\mathrm{e}}$ is the total current operator, from its very definition. It includes thus the convective current, $Z_{\mathrm{i}} u$, due to the motion of the screening cloud with the impurity as well as the friction current due to the scattering of the free carriers by the moving impurity. Let us use $J_{\mathrm{e}}^{(2)}$ for this latter term, which would be obtained for instance in a Boltzmann equation analysis. We have :

$$
J_{\mathrm{e}}=Z_{\mathrm{i}} u+J_{\mathrm{e}}^{(2)} .
$$

This distinction between the two kinds of currents will be of importance in defining the direct electrostatic force.

\subsection{FORCE ON A FIXED IMPURITY IN THE PRESENCE OF} AN ELECTRIC FIELD. - In order to establish (2), we must compare (16) with the total force $F_{\mathrm{i}}$ acting on the impurity when it is held fixed in the presence of an electric field. This force is the sum of that on the bare ion and the force exerted by the electrons. We write it as :

$$
F_{\mathrm{i}}=Z_{\mathrm{i}}|e| E+\langle F\rangle
$$

where $F$ is the electron-impurity force operator defined in (15). We note that $F$ is the total force exerted by the electrons on the impurity, whether due to the electrons in the screening cloud or to the scattering of free electrons off the impurity. At the moment, we do not distinguish between these different mechanisms and therefore there is no ambiguity in the definition of $F$.

In order to calculate $\langle F\rangle$ we use the standard linear response theory. The result is again exact to first order in the electric field $\mathbf{E}$. We assume that $\mathbf{E}$ is introduced adiabatically from $t=-\infty$ to $t=0$ so that the Hamiltonian describing the electron system is now :

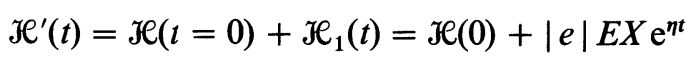

where $\mathcal{H e}(0)$ and $X$ are defined by (5) and (7) respectively and $\eta \rightarrow+0$. The eigenvalues of $\mathscr{H}(0)$ and diagonal elements of $\rho_{0}$ are $E_{m}$ and $\left(\rho_{0}\right)_{m m}=v_{m}$ respectively as defined in (13). The friction force on the impurity is :

$$
\langle F\rangle=\operatorname{Tr}(\delta \rho F)=\sum_{m n} \delta \rho_{m n} F_{n m} .
$$

Using Liouville's equation :

$$
\text { i } \delta \dot{\rho}=\left[\mathcal{H}^{\prime}(t), \rho\right]
$$

it is straightforward to show that to first order in the applied field :

$$
i \delta \rho(0)=\int_{-\infty}^{0} \mathrm{e}^{i \mathcal{J E}(0) t}\left[\mathcal{H E}_{1}(t), \rho_{0}\right] \mathrm{e}^{-i \mathcal{J e}(0) t} \mathrm{~d} t
$$

so that the matrix elements of $\delta \rho$ are now :

$$
\delta \rho_{n m}(0)=\frac{v_{m}-v_{n}}{\left(E_{m}-E_{n}\right)+i \eta}|e| E X_{n m} .
$$

On comparing (22) and (20) with (16), we see that the total interaction force $\langle F\rangle$ in the presence of an electric field is related to the total current $J_{\mathrm{e}}$ induced by the moving impurity :

$$
\langle F\rangle=-|e| E\left(\frac{J_{\mathrm{e}}}{u}\right)_{\mathbf{E}=0} .
$$

This expression is exact as it was derived on very general arguments, without any handwaving interpretations.

The net force on the impurity is given by (18) and according to the definition (17), we may write it as :

$$
F_{\mathrm{i}}=|e| E\left[Z_{\mathrm{i}}-\left(\frac{J_{\mathrm{e}}}{u}\right)_{\mathbf{E}=0}\right]=-|e| E\left(\frac{J_{\mathrm{e}}^{(2)}}{u}\right)_{\mathbf{E}=0}
$$

We have thus clarified and demonstrated the relation (2). We see that we have the choice between two equivalent interpretations :

(i) either we include in $J_{\mathrm{e}}$ the convective current of the screening cloud : we must then include in $F_{\mathrm{i}}$ the electrostatic force on the bare ion,

(ii) or we only keep in $J_{\mathrm{e}}$ the current due to the scattering of free electrons by the impurity, disregarding the transport of the screening carriers : then, we must exclude the bare electrostatic force.

Put another way, either we treat the impurity as globally neutral everywhere (both in $F_{\mathrm{i}}$ and in $J_{\mathrm{e}}$ ): this is the view advocated by Bosvieux and Friedel. Or, instead we single out the bare ion everywhere (including the bare electrostatic force in $F_{\mathrm{i}}$ as well as the screening cloud convection in $J_{\mathrm{e}}$ ). Choosing between these two attitudes is mostly a matter of taste.

One last remark : we carried the preceding dis- 
cussion for one impurity. Actually, it remains perfectly valid for a system of $n_{\mathbf{i}}$ impurities as long as they all move with the same velocity $u ; V$ is then the total electron-impurity interaction. The net current $J_{\mathrm{e}}$ and the net force $F_{\mathrm{i}}$ are both multiplied by $n_{\mathrm{i}}$ so that (2) remains true.

2.3 SPECIAL CASE WHERE THE ELECTRONS EXPERIENCE FRICTION ONLY ON THE IMPURITIES. - In the most general case the force operator $F$ can be written as :

$$
F=-\frac{\partial V}{\partial x}=\sum_{l} \frac{\partial V}{\partial x_{l}}=i[P, V]
$$

where $P=\sum_{l} p_{l}$ is the $x$-component of the total momentum of the electron gas. We now assume that the impurity potential provides the only friction mechanism acting to slow down the electrons (no thermal scattering, etc.). Then $P$ commutes with the rest of the Hamiltonian and consequently :

$$
[P, V]_{m n}=[P, \operatorname{He}(0)]_{m n}=\left(E_{n}-E_{m}\right) P_{m n} .
$$

This expression is simply the statement that $F$ provides the whole momentum transfer to the electron gas :

and

$$
F=-\frac{\partial P}{\partial t}=i[P, \mathscr{H}(0)]
$$

The total electron current induced by the displacement at velocity $u$ of the impurities is accordingly :

$$
\begin{aligned}
\left(\frac{J_{\mathrm{e}}}{u}\right)_{\mathrm{E}=0} & =i \sum_{m n}\left(v_{m}-v_{n}\right) P_{m n} X_{n m} \\
& =i \operatorname{Tr} \rho_{0}[P, X]=n .
\end{aligned}
$$

Such a result was indeed to be expected : if the electrons interact only with impurities, they are dragged along as the latter are moving, hence a net current $n u$. We note that this result is independent of the impurity concentration $n_{\mathrm{i}}$ : in the absence of other friction mechanisms the electrons sit still in the frame of reference moving along with the impurities, whatever their number.

In this special case the force exerted by the electrons on the impurities in the electric field $E$ is from (23) :

$$
\langle F\rangle=-n|e| E
$$

$(\langle F\rangle$ is the net force, including the scattering force as well as the force on the screening cloud). Once again (30) was to be expected from simple considerations of equality of action and reaction : under steady conditions the force $-\langle F\rangle$ exerted by the impurities on the electrons must balance exactly the electrostatic force $-n|e| E$ experienced by the electron gas and (30) follows at once.
The result (30) is actually pathological in that the force $\langle F\rangle$ does not depend on the impurity concentration $n_{\mathrm{i}}$ and the force on a given impurity behaves as $1 / n_{\mathrm{i}}$. This unphysical feature arises from our assumption that there is strictly no friction except on the impurities. Then a single moving impurity is in principle able to drag along the whole electron gas : conversely the net electrostatic force on the electron gas is transferred to the impurities, however few they are. These features disappear as soon as other friction mechanisms are taken into account : the force $\langle F\rangle$ is then proportional to $n_{\mathfrak{i}}$, as any extensive quantity should be. We discussed this point at some length to show the internal consistency of our approach and to emphasize the pathological nature of the case where the impurities provide the only friction mechanism on the electrons.

\subsection{ReLATION BETWEen THE EFFECTIVE VALENCE} AND THE SPECIFIC RESISTIVITY. - We now return to the general case where other friction mechanisms act in addition to impurity scattering. In order to connect $F_{\mathrm{i}}$, the net force on a given impurity, to its specific resistivity, we use a phenomenological argument. We divide the electrons in two classes, (i) those that build the screening cloud, denoted by the index 1 , form a total charge $-n_{\mathrm{i}} Z_{\mathrm{i}}|e|$, (ii) those that are free to reach the end of the crystal and which therefore give rise to electrical conduction, these we denote by the index 2.

For one impurity, the bound electrons 1 are subject to :

- the electrostatic force $-Z_{\mathrm{i}}|e| E$,

- the elastic force $f_{2}$ exerted by the impurity ion which bounds the screening charge to the impurity,

- the force $g$ exerted on the screening electrons 1 by the moving free electrons 2 .

The mechanical balance of these forces implies that :

$$
-Z_{\mathbf{i}}|e| E+f_{2}+g=0 .
$$

Similarly the electrons 2 ( $n_{0}$ in number) experience : - the force $-n_{0}|e| E$ from the direct action of the electric field,

- the force $n_{\mathrm{i}} f_{1}$ due to the bare impurities,

- the force $-n_{\mathrm{i}} g$ due to the interaction with bound electrons 1 .

The net friction force on the moving electrons is $n_{\mathrm{i}}\left(f_{1}-g\right):$ it is that combination that controls the impurity resistivity.

Now, what we found in section 2.2 was the total force exerted by all the electrons (whether bound or free) on one impurity ion. Owing to the equality of action and reaction, this force is :

$$
\langle F\rangle=-\left(f_{1}+f_{2}\right) \text {. }
$$


On combining (32) with (31) we see that we can write the net friction force on the moving electrons 2 as :

$$
n_{\mathrm{i}}\left(f_{1}-g\right)=-n_{\mathrm{i}}\left[\langle F\rangle+Z_{\mathrm{i}}|e| E\right]=-n_{\mathrm{i}} F_{\mathrm{i}} .
$$

It follows that $n_{\mathrm{i}} F_{\mathrm{i}}$, the total force on the impurities, is opposite to the net friction force on the moving electrons, irrespective of the presence of a screening cloud. It is to be noticed that the proof of this result does not make use of the thermodynamic relation (2).

The final step of the argument is now straightforward : $n_{\mathrm{i}}\left(f_{1}-g\right)$ represents the friction force on the free electrons due to the impurities. On the other hand, the total friction force due to the impurities and all other scattering mechanisms must balance the electrostatic force $-n_{0}|e| E$ on the free electrons and therefore it is equal to $n_{0}|e| E$. Assuming that the Matthiessen's rule is valid, we can write that the contribution of each scattering mechanism to the resistivity is proportional to the friction force it applies to the free electrons. We can then write the ratio of the impurity resistivity to the total resistivity $\rho$ as :

$$
\frac{\rho_{\mathrm{i}} n_{\mathrm{i}}}{\rho}=\frac{n_{\mathrm{i}}\left(f_{1}-g\right)}{n_{0}|e| E} .
$$

From (33) it follows that the net force on a given impurity is :

$$
F_{\mathrm{i}}=-n_{0}|e| E \frac{\rho_{\mathrm{i}}}{\rho}
$$

where $\rho=\rho_{0}+\rho_{\mathrm{i}} n_{\mathrm{i}}$ is the total resistivity of the system. We thereby demonstrate the validity of (4), together with the fact that the so-called direct electrostatic force $F_{\mathrm{es}}$ vanishes, as emphasized by Bosvieux and Friedel.

3. Thermodynamic analysis. - The above results can also be derived in a phenomenological way which stresses their physical origin. We consider a solution containing $n_{\mathrm{i}}$ impurities of valence $Z_{\mathrm{i}}$ and $n=n_{0}+Z_{\mathrm{i}} n_{\mathrm{i}}$ electrons per unit volume. We assume that the ions move slowly, carrying their screening cloud adiabatically along with them. We shall thus consider as the basic entity the screened neutral impurity interacting with the free electrons $\left(n_{0}\right.$ in number). We define the thermodynamic forces $X_{\mathrm{i}}$ and $X_{\mathrm{e}}$ acting on a neutral impurity and a free electron respectively :

$$
\left\{\begin{array}{l}
\mathbf{X}_{\mathrm{i}}=-\nabla \mu_{\mathrm{i}}=-k T \frac{\nabla n_{\mathrm{i}}}{n_{\mathrm{i}}} \\
\mathbf{X}_{\mathrm{e}}=-\nabla \mu_{\mathrm{e}}=-\nabla \mu^{\prime}-|e| \mathbf{E}
\end{array}\right.
$$

where $\mu_{\mathrm{i}}=k T \ln n_{\mathrm{i}}$ and $\mu_{\mathrm{e}}=\mu^{\prime}\left(n_{\mathrm{i}}\right)-|e| V$ are their chemical potentials. The solid solution is assumed very dilute so that no activity coefficient appears in the definition of $\mu_{\mathrm{i}}$ and the term $\mu^{\prime}\left(n_{\mathrm{i}}\right)$ exhibits the dependence of $\mu_{\mathrm{e}}$ on the impurity concentration.

According to the thermodynamics of irreversible processes, the fluxes of impurity atoms and of electrons are respectively (9) :

$$
\left\{\begin{array}{l}
\mathbf{J}_{\mathrm{i}}=-L_{\mathrm{ii}} \nabla \mu_{\mathrm{i}}-L_{\mathrm{ie}} \nabla \mu_{\mathrm{e}} \\
\mathbf{J}_{\mathrm{e}}=-L_{\mathrm{ei}} \nabla \mu_{\mathrm{i}}-L_{\mathrm{ee}} \nabla \mu_{\mathrm{e}}
\end{array}\right.
$$

where $L_{\mathrm{ei}}=L_{\mathrm{ie}}$ from Onsager's relations. The coefficients $L_{\mathrm{ee}}$ and $L_{\mathrm{ii}}$ are related to the electric resistivity $\rho$ of the alloy and to the diffusion coefficient $D_{\mathrm{i}}$ of the impurity by the equations :

$$
\left\{\begin{array}{l}
L_{\mathrm{ee}}=\frac{1}{\rho e^{2}} \\
L_{\mathrm{ii}}=\frac{n_{\mathrm{i}}}{k T} D_{\mathrm{i}} .
\end{array}\right.
$$

In order to identify the coefficients $L_{\mathrm{ie}}$ and $L_{\mathrm{ei}}$ we examine more closely the friction processes leading to a stationary current in the system. The friction force $\varphi_{e}$ acting on the electrons of a unit volume of the alloy can be written in a linear approximation :

$$
\boldsymbol{\varphi}_{\mathrm{e}}=-n_{0} \alpha \mathbf{u}_{\mathrm{e}}-n_{0} n_{\mathrm{i}} \beta\left(\mathbf{u}_{\mathrm{e}}-\mathbf{u}_{\mathrm{i}}\right)
$$

where $\mathbf{u}_{\mathrm{e}}$ and $\mathbf{u}_{\mathrm{i}}$ are the drift velocity of electrons and impurities with respect to the lattice ; $\alpha$ and $\beta$ are the friction coefficients of electrons on the lattice and on an impurity respectively. In the same way the friction force $\varphi_{i}$ on the $n_{i}$ impurities contained in a unit volume is :

$$
\boldsymbol{\varphi}_{\mathrm{i}}=n_{0} n_{\mathrm{i}} \beta\left(\mathbf{u}_{\mathrm{e}}-\mathbf{u}_{\mathrm{i}}\right)-n_{\mathrm{i}} \gamma \mathbf{u}_{\mathrm{i}} .
$$

The first term in this expression is the friction force of free electrons on the perturbation due to the presence of impurities. The coefficient $\gamma$ expresses the mean friction force experienced by the diffusing atom during its diffusion excursions.

When a stationary state is reached the net forces $n_{\mathrm{i}} \mathbf{X}_{\mathrm{i}}+\varphi_{\mathrm{i}}$ on impurity atoms and $n_{0} \mathbf{X}_{\mathrm{e}}+\varphi_{\mathrm{e}}$ on free electrons vanish. It is then possible to solve for the velocities $\mathbf{u}_{\mathbf{i}}$ and $\mathbf{u}_{\mathrm{e}}$ and to obtain the fluxes $\mathbf{J}_{\mathbf{i}}$ and $\mathbf{J}_{\mathrm{e}}$ as functions of the thermodynamic forces $\mathbf{X}_{\mathrm{i}}$ and $\mathbf{X}_{\mathrm{e}}$.

This procedure leads to the following identification of the phenomenological coefficients :

$$
L_{\mathrm{ii}}=\Delta^{-1} n_{\mathrm{i}}\left(\alpha+n_{\mathrm{i}} \beta\right) ; L_{\mathrm{ee}}=\dot{\Delta}^{-1} n_{0}\left(\gamma+n_{0} \beta\right)
$$

where

$$
\Delta=\left(\alpha+n_{\mathrm{i}} \beta\right)\left(\gamma+n_{0} \beta\right)-n_{\mathrm{i}} n_{0} \beta^{2} .
$$

As we consider a very dilute solution we assume that $n_{\mathrm{i}} \beta$ is much smaller than $\alpha$; on the other hand the friction coefficient $\gamma$ of an atom on the lattice is very much larger than the friction coefficient $n_{0} \beta$ on 
the electron gas. This allows us to neglect the second term in (42) and we obtain :

$$
\begin{aligned}
\rho & =\frac{\alpha+n_{\mathrm{i}} \beta}{n_{0} e^{2}} \\
D_{\mathrm{i}} & =\frac{k T}{\gamma} \\
\mathbf{u}_{\mathrm{i}} & =-\frac{D_{\mathrm{i}}}{k T} \cdot \frac{\beta}{\rho e^{2}}|e| \mathbf{E} .
\end{aligned}
$$

From (43) we identify the specific resistivity $\rho_{\mathrm{i}}$ of the solute :

$$
\rho_{\mathrm{i}}=\frac{\beta}{n_{0} e^{2}}
$$

and from (45) the usual result for the effective valence of the solute is recovered :

$$
Z_{\mathrm{i}}^{*}=-\frac{n_{0} \rho_{\mathrm{i}}}{\rho}
$$

An equivalent procedure to derive this expression has been used previously by Flynn [10] and Turban [5].

The fact there is no contribution to $Z_{i}^{*}$ of a direct electrostatic force follows from our considering perfectly screened ions.
4. Conclusion. - In this paper we have shown that an analysis of the friction forces acting on the electron gas leads to the well known relation (15) between the effective valence and the specific resistivity of an impurity. Through a linear response formalism we have also shown the microscopic equivalence between the definitions (1) and (2) established in the framework of the thermodynamics of irreversible processes. Our treatment can be largely extended to a substitutional impurity. In particular the electrostatic force vanishes because there is no net transport of electric charge when a permutation between an impurity and a vacancy occurs. There is a fundamental question regarding the value of $\rho_{\mathrm{i}}$ to use in eq. (4). For a slowly diffusing impurity in a liquid for instance, it seems likely that the impurity is accompanied by its neighbours during an elementary displacement so that the specific resistivity $\rho_{\mathrm{i}}$ determined in a standard resistivity measurement is relevant to the diffusion problem. In case of a rapidly diffusing impurity in a.solid or a liquid, it is not clear if $\rho_{\mathrm{i}}$ is actually relevant because it contains a contribution of the relaxed neighbours as well as of the jumping ion itself. Although some attempts [11] to solve this problem have been made, it is necessary to perform a complete dynamical analysis of the jump process in presence of the electric field in order to identify the forces responsible for the drift velocity of the solute.

\section{References}

[1] Nguyen van Doan, Thesis, Orsay (1970).

[2] Huntington, H. B., Grone, A. R., J. Phys. \& Chem. Solids 20 (1961) 76.

[3] Fiks, V. B., Sov. Phys. Solid State 1 (1959) 14.

[4] Bosvieux, C., Friedel, J., J. Phys. \& Chem. Solids 23 (1962) 123.

[5] Turban, L., Thesis, Nancy (1975).

[6] Kumar, P., Sorbello, R. S., Paper presented at International Conference on low temperature diffusion and applications to thin films, I.B.M., Yorktown Heights (1974).
[7] Schaich, W. L., To be published in Phys. Rev. B.

[8] ICHE, G., Nozières, P., To be published in Phys. Rev. B.

[9] Adda, Y., Philibert, J., La diffusion dans les solides. Bibliothèque des Sciences et Techniques Nucléaires (P.U.F.) 1966.

[10] Flynn, C. P., Point defects and diffusion (Oxford University Press, London) 1972.

[11] Sorbello, R. S., J. Phys. \& Chem. Solids 34 (1973) 937. 